\title{
NOUVELLe
}

\section{Élongation de l'embryon : un mouvement cellulaire aléatoire}

Équipe d'Olivier Pourquié,

Programme de biologie du développement et cellules souches, Institut de génétique et de biologie moléculaire

et cellulaire (IGBMC),

67404 Illkirch Cedex, France

benazera@igbmc.fr
> Au cours de son développement, l'embryon de vertébrés subit une série de changements morphogénétiques nécessaires à la différenciation correcte de toutes les structures de l'individu adulte. Durant les premières étapes de la vie, l'embryon est constitué d'un groupe de cellules organisées en une structure symétrique ronde. Puis, lors de la gastrulation, étape de mise en place des trois feuillets embryonnaires (endoderme, mésoderme et ectoderme), cette symétrie est brisée et l'embryon s'allonge selon l'axe antéropostérieur (ou tête/queue) (Figure 1A). La formation du corps se produit de manière progressive en commençant d'abord par la tête, puis le cou, le thorax et enfin l'abdomen. Les ébauches de ces différentes structures sont progressivement formées à partir de la partie postérieure de l'embryon appelée bourgeon caudal (Figure IB).

\section{Comportements migratoires \\ des cellules au cours du processus \\ d'élongation embryonnaire}

Le phénomène d'élongation embryonnaire est généralement décrit comme résultant de mouvements cellulaires spécifiques appelés mouvements de convergence-extension [1]. La convergence-extension se caractérise par l'insertion de cellules orientées perpendiculairement à l'axe antéropostérieur de l'embryon. Ces mouvements cellulaires vont permettre de transformer un tissu large et court en un tissu long et fin (allongement). Ils sont très bien décrits chez l'embryon de poisson, de grenouille et de poulet à un jour de développement (Figure 1A) [1-3]. Un jour plus tard, alors que l'allongement de l'embryon se poursuit, le taux de convergence tissulaire a diminué de manière significative. Un autre mécanisme cellulaire doit donc lui être substitué pour expliquer l'élongation à ce stade. Dans une étude récente, nous avons utilisé l'embryon de poulet pour élucider cette apparente contradiction [4]. Nous avons tout d'abord procédé à l'ablation de différentes régions dans la partie postérieure de l'embryon et mesuré le taux d'élongation. Cette approche nous a permis de mettre en évidence le rôle central de la partie postérieure du mésoderme présomitique (psm) dans ce mécanisme de morphogenèse. Le psm est constitué de deux bandes de tissu mésenchymateux situées de part et d'autre du tube neural. Pour étudier les comportements migratoires des cellules du psm nous les avons marquées grâce à une technique de transfection in vivo, par électroporation [5], du gène codant pour la GFP (green fluorescent protein). Nous avons alors observé que ces cellules sont beaucoup plus mobiles dans la partie postérieure du psm que dans sa partie antérieure. Autrement dit, les cellules situées près du bourgeon caudal bougent plus activement et librement que celles qui sont situées dans la partie antérieure du psm, qui sont davantage compactées et quasi-immobiles. De manière intéressante, en analysant la direction vers laquelle s'orientaient ces mouvements cellulaires, nous avons observé que les cellules se déplaçaient majoritairement vers la partie postérieure de l'embryon, dans la même direction que l'allongement du tissu.
Mouvement aléatoire des cellules proches du bourgeon caudal

Se posait alors la question de l'origine de ces mouvements: sont-ils dus aux mouvements actifs des cellules ou bien à un déplacement de l'espace dans lequel elles évoluent? Par analogie, la question est la même que celle qui consiste à se demander si le déplacement d'une personne marchant sur un bateau voguant à vive allure est majoritairement dû au déplacement du bateau ou à celui de la personne. Pour répondre à cette question nous avons donc soustrait les mouvements du tissu aux mouvements des cellules. Nous avons utilisé comme repère un élément de la matrice extracellulaire, la fibronectine, qui ne possède pas de propriété motrice [6]. Nous avons été surpris de constater qu'après cette soustraction, les mouvements cellulaires ont perdu toute orientation précise mais que cependant le gradient de mouvement (fort en postérieur et faible en antérieur) était toujours présent. II existe donc un gradient de motilité cellulaire non directionnelle au sein du mésoderme présomitique. Ce phénomène ressemble à de la diffusion moléculaire: des molécules soumises à un gradient de chaleur vont se disperser préférentiellement là ou la température est la plus importante. La diffusion cellulaire étant plus importante à proximité du bourgeon caudal, cela peut expliquer que l'embryon s'allonge majoritairement vers sa partie postérieure. Pour s'assurer de ce résultat (la plupart des processus de morphogenèse sont décrits comme étant le fruit de migrations cellulaires dirigées et non 


\section{A Élongation à environ un jour de développement}

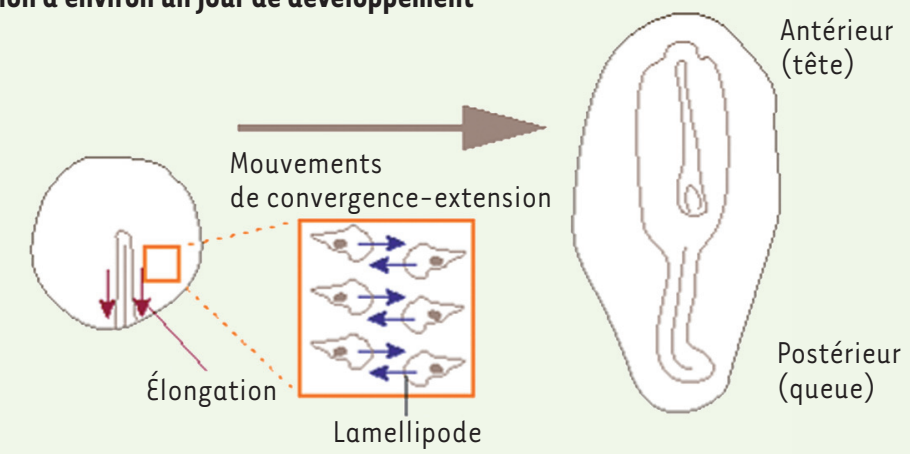

B Élongation à environ deux jours de développement

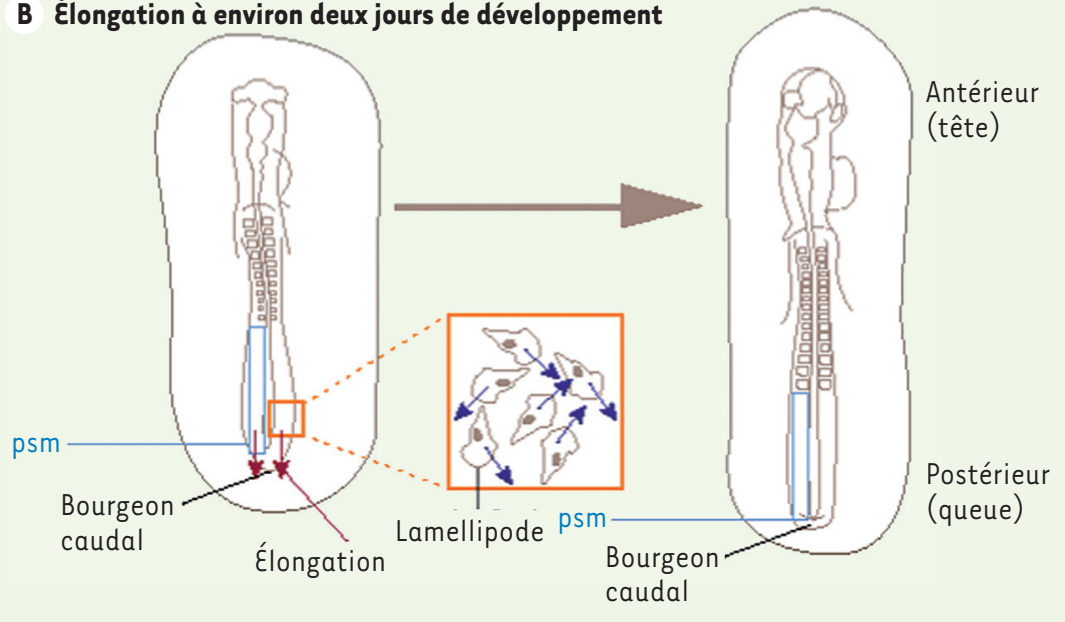

aléatoires) nous avons vérifié l'absence de biais directionnel au niveau de la morphologie des cellules. Les lamellipodes (protrusions principales des cellules leur permettant de se mouvoir) étant généralement orientés dans le sens du mouvement cellulaire, nous avons analysé leur direction par rapport à l'axe de l'embryon. À l'inverse des cas de migration dirigée, nous n'avons pas pu mettre en évidence d'orientation particulière de ces protrusions dans le psm postérieur (Figure 1, encarts oranges), confirmant ainsi le mouvement aléatoire des cellules.

\section{Rôle-clé de la signalisation FGF dans la migration cellulaire}

Nous avons ensuite cherché à identifier les signaux qui pouvaient être à l'origine du gradient de mouvements aléatoires dans le psm. La signalisation FGF (fibroblast growth factor) était un très bon candidat pour rendre compte de cet effet et ceci pour deux raisons majeures. D'abord cette signalisation intervient déjà sous forme de gradient dans le psm, où elle joue un rôle important dans la formation des structures adultes comme les vertèbres $[7,8]$. Ensuite, cette signalisation a été impliquée dans le mouvement des cellules du mésoderme et en particulier du psm [9]. Nous avons effectué des expériences de perte et de gain de fonction de la signalisation FGF afin d'évaluer son rôle dans l'établissement du gradient de motilité aléatoire et dans l'élongation. La perte de fonction cause l'inhibition des mouvements dans tout le psm alors que le gain de fonction provoque l'induction des mouvements cellulaires dans le psm antérieur (là où les cellules sont immobiles dans la condition contrôle). Dans les deux cas l'élongation de l'embryon ralentit. Cela paraît surprenant mais peut s'expliquer facilement: le gradient de mouvement est nécessaire à une dif-
Figure 1. Vues schématiques des différents modes d'élongation axiale chez l'embryon de poulet. A. Élongation à environ un jour de développement. Passage d'un stade $3 \mathrm{HH}$ (Hamburger et Hamilton [10]) à un stade $6 \mathrm{HH}$. Les cellules qui convergent (rectangle orange) entraînent la convergence du tissu embryonnaire et l'extension/élongation du blastoderme (flèches rouges). B. Élongation à environ deux jours de développement. Passage d'un stade $11 \mathrm{HH}$ à un stade $13 \mathrm{HH}$. Les cellules qui migrent de façon non directionnelle dans la partie postérieure du psm (rectangle orange) entraînent l'allongement postérieur de l'embryon (flèches rouges).

fusion localisée des cellules permettant l'allongement postérieur du tissu. $\varepsilon$ n effet, si les cellules ne bougent plus, le tissu ne peut pas grandir. En revanche, si toutes les cellules bougent de la même façon, le tissu ne s'étale plus postérieurement mais dans toutes les directions. Ces résultats nous montrent que le gradient de motilité joue un rôleclé dans l'efficacité de l'élongation. La signalisation FGF affecte à la fois la motilité et la prolifération cellulaires. Afin de distinguer l'implication de l'une et l'autre, nous avons bloqué indépendamment la motilité et la prolifération à l'aide d'inhibiteurs spécifiques. Pour des temps de traitement courts, le blocage des mouvements cellulaires affecte l'élongation alors que l'inhibition de la prolifération n'a pas d'effet, indiquant que l'action du FGF sur l'élongation passe principalement par son rôle sur la migration cellulaire.

$\varepsilon n$ résumé, notre étude met en évidence que l'allongement progressif du corps de l'embryon fait intervenir le gradient de mouvements aléatoires observé dans le psm. L'apport de nouvelles cellules (recrutement dans le psm et prolifération) permet au système de maintenir son allongement postérieur. Il semble donc qu'à ces stades du développement les cellules ne possèdent pas d'information de direction de migration mais peuvent interpréter cependant un gradient 
de signal en se déplaçant plus ou moins, sans direction. La directionnalité de l'élongation se mettrait en place via un phénomène de diffusion différentielle, mais aussi en réponse aux contraintes physiques du psm et des tissus adjacents. À l'heure actuelle, nous ne savons que peu de choses sur ces contraintes. II semble donc que la compréhension des propriétés physiques de ce tissu s'avérera une étape incontournable pour comprendre les phénomènes de morphogenèse et constituera certainement un des enjeux importants en biologie du développement durant ces prochaines années. $\diamond$

Elongation of the embryo results

from random cell motion
CONFLIT D'INTÉRÊTS

Les auteurs déclarent n'avoir aucun conflit d'intérêts concernant les données publiées dans cet article.

\section{RÉFÉRENCES}

1. Keller R. Cell migration during gastrulation. Curr Opin Cell Biol 2005 ; 17 : 533-41.

2. Voiculescu O, Bertocchini F, Wolpert L, et al. The amniote primitive streak is defined by epithelial cell intercalation before gastrulation. Nature 2007 ; 449: 1049-52.

3. Lawson A, Schoenwolf GC. Cell populations and morphogenetic movements underlying formation of the avian primitive streak and organizer. Genesis 2001; 29: 188-95.

4. Bénazéraf $B$, Francois $P$, Baker RE, et al. A random cell motility gradient downstream of FGF controls elongation of an amniote embryo. Nature 2010 ; 466: 248-52.

5. limura T, Pourquie 0. Manipulation and electroporation of the avian segmental plate and somites in vitro. Methods Cell Biol 2008; 87 : 257-70.

\section{NOUVELL}

\section{Vers une cartographie des tensions mécaniques intracellulaires?}

Virgile Viasnoff
6. Zamir EA, Czirok A, Cui C, et al. Mesodermal cell displacements during avian gastrulation are due to both individual cell-autonomous and convective tissue movements. Proc Natl Acad Sci USA 2006 ; 103: 19806-11.

7. Dubrulle J, McGrew MJ, Pourquie O. FGF signaling controls somite boundary position and regulates segmentation clock control of spatiotemporal Hox gene activation. Cell $2001 ; 106: 219-32$.

8. Dubrulle J, Pourquie 0. fgf8 mRNA decay establishes a gradient that couples axial elongation to patterning in the vertebrate embryo. Nature 2004 ; 427 : 419-22.

9. Delfini MC, Dubrulle J, Malapert P, et al. Control of the segmentation process by graded MAPK/ERK activation in the chick embryo. Proc Natl Acad Sci USA 2005; 102 : 11343-8.

10. Hamburger V, Hamilton HL. A series of normal stages in the development of the chick embryo.1951. Dev Dyn $1992 ; 195: 231-72$.
ESPCI ParisTech, CNRS UMR 7083 et MBI, National University of Singapore, 10, rue Vauquelin, 75005 Paris, France. virgile.viasnoff@espci.fr

mécaniques externes qui pourraient potentiellement être intégrées au niveau de la membrane cellulaire ou du nucléosquelette afin d'activer le programme de transcription génétique approprié.

\section{Mesure des forces exercées}

par les cellules sur leur environnement La sollicitation mécanique peut être elle-même exercée par la cellule afin de sonder les propriétés microrhéologiques de son environnement. Les cellules souches mésenchymateuses (MSC) peuvent par exemple se différencier en neurones, myoblastes ou ostéoblastes selon la rigidité de leur substrat de culture [4]. La mesure des déformations locales de substrats élastiques a permis d'évaluer à plusieurs dizaines de $\mathrm{nN}$ (nanonewtons) les forces exercées sur la matrice extracellulaire par des cellules lors de leur migration [5]. L'existence de points 